\title{
Culture Confronts Nature in the Dialectical World of the Tropics
}

\author{
BILLIE JEAN ISBELL \\ Department of Anthropology \\ Cornell University \\ Ithaca, New York 14853
}

\begin{abstract}
Av VENI AND URTON are to be congratulated for organizing a conference that, for the first time, has brought together scholars from various disciplines to discuss the ethnoastronomy and archaeoastronomy of the American tropics. We have had to converse across disciplinary boundaries and I hope that the endeavor has clarified some of the concepts, methods, and perspectives of the different specialities represented.

As an anthropologist, I would like to suggest that the tropics provide a perceptual environment that promotes and enhances a particular 'science of the concrete, ${ }^{1}$ whereby perceived order in the environment is the basis for systems of classifications, epistemological structures, and cosmologies. In the American tropics, the science of the concrete takes on a particular character that results in epistemologies founded in what I will call dialectical, reversible dualism.

The native philosophers of the indigenous societies under discussion engage in the study of the nature and limits of knowledge. Their epistemological reflections are embedded in religious and ritual practices. Moreover, the native philosophers, who are usually shamans or astronomer-priests, use methods and metaphorical language that are unfamiliar to us. More importantly, the logic that underlies these systems of knowledge is dialectical rather than rationalistic. As Roy Wagner has pointed out in The Invention of Culture, the anthropological definition of the concept of dialectic refers to
\end{abstract}

a tension or dialogue-like alternation between two conceptions or viewpoints that are simultaneously contradictory and supportive of each other. 
As a way of thinking, a dialectic operates by exploiting contradictions against a common ground of similarity, rather than by appealing to consistency against a common ground of differences, after the fashion of rationalistic or 'linear' logic. ${ }^{2}$

Examples may help to clarify the two types of logic. In Western science, taxonomies and typologies employ the principle of similarity against a common ground of differences. The underlying assumption of rationalistic logic is linear causality. In sharp contrast, dialectical logic focuses upon simultaneous interdependence and contradiction. Linear causality is not assumed. For example, the most widespread dialectical concept in the American tropics is the Axis Mundi. While the cultural contents differ, the necessary tension is maintained between the opposed elements of the upperworld and the underworld. The interdependence is such that one cannot be defined without reference to the other.

By reversible dualism, I mean a logical process whereby the definition of one of the polar opposites must be derived from the view or position of the other. For example, the structure of the relationship between the polar opposites of the Axis Mundi necessarily means that one, the upperworld, must be defined by viewing it from the underworld. This principle of reversible dualism applies more generally, and I argue that astronomical phenomena in the American tropics are perceived as dual, dialectical pairs. One is necessarily the vantage point for the cultural definition of the other and vice versa. Therefore, time is perceived as a dialectical tension between two interdependent, but contradictory, elements. In part, this is due to the structural relationships that pertain between solar zenith and nadir phenomena and significant seasonal changes in weather, fauna, and flora. In turn, observable celestial periodicities - such as the phases and positions of the moon and the paths of various constellations - are sought that fit the epistemological structures that explain seasonal changes. For example, the path of the Pleiades is important everywhere in the American tropics for announcing seasonal changes. However, because the predictable appearance and disappearance of the constellation occurs with different periods throughout the American tropics, the significance differs from culture to culture. If I am correct about the structure of epistemologies in the American tropics, the Pleiades should always be opposed to some other celestial body in a dialectical, reversible relationship.

I shall discuss the structural similarities of the various native cosmologies as well as the culture-specific differences that have come to light in this conference. But first, I would like to make an observation 
about the nature of the science of the concrete. Awareness of the regularity and structure of various naturally occurring periodicities provide the schemata ${ }^{3}$ for verifying epistemologies in every culture. I suggest, however, that the structural peculiarities of the observable periodicities in the tropics result in a number of shared epistemological features.

Most significant is the fact that, in the tropics, celestial bodies move on straight tracks, rather than around a fixed point in the sky (the north and south celestial poles). ${ }^{4}$ The perceptual consequence of this phenomenon is that the sky is divided into two halves. Moreover, as Urton has explained, the point of observational orientation is the movement of celestial bodies in relation to the observer's own fixed locality, rather than a fixed celestial pole. ${ }^{5}$ Aveni has shown that all an observer needs are simple devices, such as crossed sticks directed to the horizon or

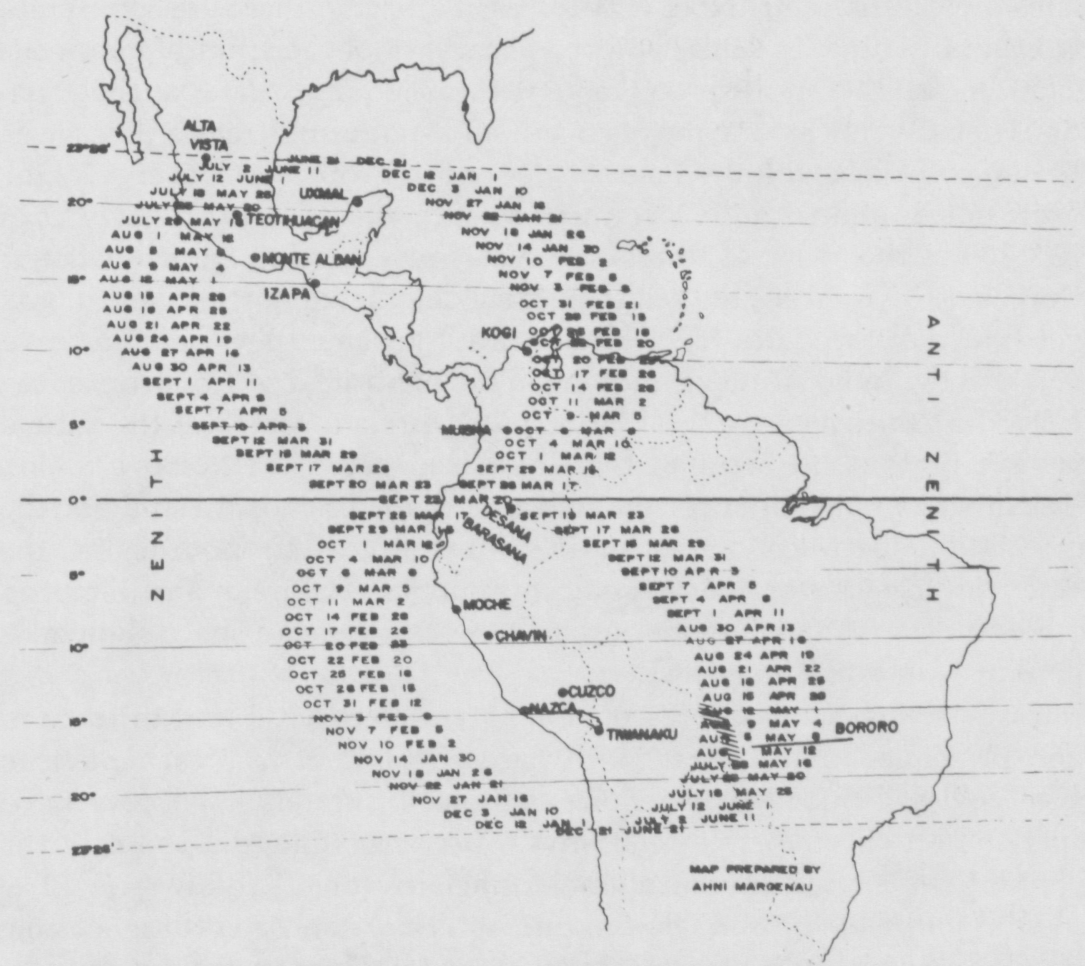

FIgURE 1. Zenith and nadir (antizenith) dates taken from the American Ephemeris and Nautical Almanac for 1980, correct for other years \pm 2 days. 
sighting tubes and gnomons oriented to the zenith, to make accurate astronomical and calendrical calculations and predict the approach of the solstices, the equinoxes, the cycles of Venus, and the zenith passages of the sun. ${ }^{6}$

Observed astronomical phenomena in tropical America play a large role in the development of epistemological structures. Specifically, the vertical orientation to the sky of an observer who is the fixed center around which celestial bodies move promotes epistemologies that have dual, symmetric, and reversible structures similar to those found in spatiotemporal structures of the native astronomy. In order to delineate a few of the similarities of the structures of space, time, and cosmologies, I will begin with an examination of zenith and nadir solar phenomena. In FIGURE 1, I have attempted to depict the symmetry and reversibility of zenith and nadir solar passage dates by drawing them in the shape of a figure eight. The zenith dates for each latitude are on the left; the nadir dates are on the right. Note that for each latitude, there are two zenith dates and two nadir dates, except at the lines of the tropics and at the equator. Zenith and nadir converge with the equinox dates at the equator. They diverge as one moves north and south until zenith passage of the sun coincides with the June solstice at the Tropic of Cancer $\left(23^{\circ} 26^{\prime}\right)$ in the north, and with the December solstice at the Tropic of Capricorn $\left(23^{\circ} 26^{\prime}\right)$ in the south. The relationship is reversed for the nadir dates. Moreover, if you trace the zenith passage dates from north to south, you will find that the dates for the nadir are the same (plus or minus two days) for the same latitudes, moving in the opposite direction from south to north. Consequently, the spatiotemporal relationships of the annual passage of the sun are like two interlaced cords, to borrow Klein's Mesoamerican metaphor for the structure of the cosmos. In studying the articulation between cosmologies and native astronomies in the American tropics, we find that culture confronts nature in an attempt to apprehend the dynamic relationship between time and space shown by the zenith and nadir passages.

In reviewing the data presented at this conference, I find three common principles of organization in the structures of tropical American cosmologies. I find, however, one significant difference. All four principles reflect the dynamic relationship between time and space in the tropics. The shared organizational principles are as follows: (1) The celestial paths of the sun, moon, and stars are conceived of as cosmic forces whose multiple interactions are responsible for transitions of time, climate, the agricultural cycle, and states of human existence. A cor- 
ollary of this is that the major metaphors for cosmic order and disorder are based on principles of movement, of transition, and of reversibility, rather than on metaphors of static equilibrium, (2) these dynamic principles, based on observations of interacting cosmic forces, are replicated onto space in settlement plans and architectural and ritual space, as if the built environment of social space were a mirror reflection of the dialectical elements in the heavens, (3) finally, the most prevalent cosmological principle in the American tropics is the Axis Mundi, accompanied by what I will call a circulatory cosmology. The Axis Mundi is a world axis around which cosmological and celestial forces circulate. It is expressed metaphorically as a world tree, cosmic mountain, or a pyramid encircled or encompassed by a river, gut, an umbilical cord, or woven fabric. This vertical axis and its circulatory cosmic flow form two opposing dynamic principles, which maintain the dialectical tension essential to cosmic order.

The major difference I find is that between the structure of Barasana and Desana cosmologies and that of the cultures to the north and south. The Barasana and the Desana are located near the equator. According to Hugh-Jones and Reichel-Dolmatoff, neither place any importance on horizon observation of the annual movement of the sun. Nor do they make use of heliacal risings and settings to fix events in a calendar. They do, however, observe the zenith passage of the sun, which occurs during the equinoxes, and the annual paths of various constellations. The Pleiades, Scorpio, and Orion are among the important constellations observed as indicators of the changing seasons. Reichel-Dolmatoff makes a point that is true, I think, for both of these equatorial cultures: they are concerned with the diagnosis of the present state of affairs in the universe, not with a prognosis for the future. I would like to suggest that, at the equator, time and space conspire to give the observer the impression of being stationary at the center of the Axis Mundi, around which the universe circulates (see Hugh-Jones' excellent description of the structure of the Old and New Path of the Milky Way for details). Additionally, the dual rainy and dry seasons and the absence of a calendar based on tracking the annual movements of the sun on the horizon may intensify the perception that one is living at the center of present time and space. Consequently, the science of the concrete for cultures at the equator should be directed toward knowledge of the variations of the flora and fauna in their environments rather than precise astronomy and prediction of future events. Reichel-Dolmatoff mentions that the Muiska and the Kogi have more complex astronomical systems than do the Desana. Whether they also have a precise calendar is not clear. Fabian 
hypothesizes that the Bororo have a complex system of observations and probably a precise calendar. These three cultures are far from the equator and would provide evidence concerning the relationship between the constraints of perceptual realities and the development of epistemologies.

As one moves farther and farther north or south away from the equator, the apparent annual movement of the rising and setting of the sun on the horizon becomes greater. ${ }^{7}$ Moreover, the dates of zenith and nadir passages of the sun diverge until, at latitudes $15^{\circ}$ north and south, the four zenith and nadir dates divide the year into more or less equal segments. Coggins argues that, at Izapa, $15^{\circ}$ north, the zenith passages of the sun define the 260-day ritual calendar. Broda gives an excellent summary of current research on this question for Mesoamerica. Aveni and Urton suggest that zenith and nadir may be important for astronomical alignments and geometric forms among the lines of Nazca ${ }^{8}$ Zuidema argues that, at Tiwanaku, $16^{\circ} 33^{\prime}$ degrees south, zenith and nadir passages divide the year into more or less equal segments ${ }^{9}$-an observation he believes to have been important to cultural developments in the Andes. ${ }^{10}$ The site of Alta Vista, located at the Tropic of Cancer, provides sound evidence that the ancient astronomers of the American tropics were aware of the regularity and structure of zenith phenomena. In discussing the location and orientation of this site, Aveni states that the astronomers seemed to have been seeking the actual place where the sun turns around on its northern migration and begins its journey to its southern turn around position. ${ }^{11}$ During the two solstices, the sun slows down in its march along the horizon as it approaches its northern and southern extremes. Aveni notes that "the sun will stand perched in the zenith at noon on the longest day, the first day of summer. A shadowless moment occurs as the sun arcs over the zenith and returns to its southern realm." 12 This annual journey of the sun is calculated by watching sunrise and sunset positions on the horizons-hence, horizon astronomy, a name that Aveni has coined.

Aveni and Zuidema's recent research on the astronomy of the Incas of Cuzco, located at $13^{\circ} 50^{\prime}$ south, provides the best evidence for the significance of nadir solar passages. ${ }^{13}$ Zuidema argues that the annual cycles of the sun, the moon, and the Pleiades were the three central celestial phenomena that were correlated together in such a way that one observed event announced the other. Urton demonstrated that the same observations are possible for the coast of Peru. In both regions, the series of astronomical observations and the agricultural cycle are conjoined. 
The most significant feature of Inca astronomy is that the zenith and nadir were diametrically opposed in space and time. The zenith sunrise point on the eastern horizon and the nadir sunset on the western horizon formed the axis upon which the sun was believed to travel. Zuidema argues that nadir sunset was established by backsighting from the zenith sunrise position to form one of the ceque lines. The June solstice sunrise and the December sunset locations were likewise connected by a ceque line. The zenith and nadir dates at the latitude of Cuzco are six months apart (see Figure 1). The zenith-nadir dates were used to time the agricultural and ritual cycle. The nadir pair, August and April, are the beginning of planting and the beginning of harvest, respectively. Important rituals were celebrated on both dates. The February and October zenith dates, according to Sherbondy's ethnohistorical research, center upon rituals concerned with the control of water. ${ }^{14}$ In February, a ritual was celebrated to signify that the earth was saturated with water and that a transition into the dry season was approaching. In October, a ritual was performed to signal the end of the first irrigation cycle; this period is a transition into the rainy season. Conversely, one pair of opposite dates, the August nadir, the beginning of the agricultural cycle, and the February zenith symbolically form the Andean Axis Mundi. The earth is still believed to open up on these dates to receive offerings. These two periods, opposed in time and space, are both propitious and dangerous. It appears to me that Andean people discovered an organizing principle of dialectical, reversible dualism that ordered their time, space, and society. The cultural focus seems to be on determining transitions from one cycle or state to another: from the dry season to the rainy season; from the sun's journey to the north to its journey to the south; from periods of abundance and prosperity to periods of scarcity and poverty. These periods were announced by specific series of celestial events and mapped onto space in axial sight lines (the ceques) that were observationally reversible (i.e., zenith sunrise and antizenith sunset) and semantically dualistic. I would like to suggest that a similar structural organization might be discovered for Mesoamerica.

In Mesoamerica, the prevalence of the concept of the Axis Mundi (see Coggins' paper) and the importance of the zenith passages for regulating the agricultural cycle suggest that the nadir passages may have more significance than previously believed. Moreover, both the Andean and Mesoamerican areas of complex cultural development are at latitudes where zenith and nadir passage dates are spaced such that they can be used in similar structural organizations.

Broda points out that, at Tenochititlan, the two zenith dates were 
commemorated by important rituals in May and July. During the period in between these two dates, the sun entered the abode of the dead, which was believed to be in the north. I assume that this coincided with the sun's journey to the north, where it appeared to slow down before turning around during the June solstice to begin its journey towards its southern extreme. In addition, she discusses the coincidence of the disappearance of the Pleiades with the zenith passage of the sun on 17 May as contrasted with the nadir passage of the sun on 18 November, when the Pleiades were observed at the zenith. Thus, she argues for an "opposite symmetry" between the path of the sun and the path of the Pleiades. Climatologically, these two events were significant. The rains were announced by the disappearance of the Pleiades and the shadowless moment of zenith passage of the sun on 17 May. The dry season was heralded by the appearance of the Pleiades at the zenith and the sun at the nadir on 18 November. Therefore, one can think of the sun and the Pleiades as existing on an axis that announces the changing seasons. They form a dialectical, reversible, dual structure.

Likewise, Zuidema and Urton both argue that the multiple interactions of the zenith/nadir dates, the path of the Pleiades, and the phases and positions of the moon announce the changing seasons in Cuzco and on the coast of Peru. But whereas Broda finds an "opposite symmetry" prevailing between the course of the sun and that of the Pleiades, Zuidema and Urton find convergence. They both argue that the disappearance of the Pleiades at the end of April coincides with the nadir, which announces major transition periods. Zuidema argues that the disappearance of the Pleiades and the sun at the nadir symbolized the death of the sun, the moon, and the Pleiades. Beginning the harvest at this time in April caused Earth Mother to die as well. A period of rebirth was begun with the reappearance of the Pleiades (heliacal rise at dawn) in early June in Cuzco and on the coast of Peru. Notice that the cultural focus is on transitions and transformations of states, rather than on static moments. The logic is dialectical, not rationalistic. If we compare the interpretations of the disappearance of the Pleiades from the information given in Broda's, Zuidema's and Urton's paper, we get TABLE 1.

I have discussed these examples in some detail to illustrate that the same astronomical event (the disappearance of the Pleiades) is taken to be highly significant in both Mesoamerica and the Andes. However, this easily observable event is correlated with other highly significant events: the zenith and nadir of the sun. In order to gain further understanding of the cosmological meanings of this set of multiple observations, we have 
TABLE 1

The Disappearance of the Pleiades Interpreted

\begin{tabular}{|c|c|c|}
\hline $\begin{array}{l}\text { Multiple Astronomical } \\
\text { Events Observed }\end{array}$ & Transition Announced & Location \\
\hline $\begin{array}{l}\text { May } 17 \\
\text { Zenith sun } \\
\text { Disappearance of the Pleiades } \\
\text { ("opposite symmetry") or axis }\end{array}$ & $\begin{array}{l}\text { Onset of rains } \\
\text { Sun enters world of } \\
\text { the dead }\end{array}$ & $\begin{array}{l}\text { Tenochititlan } \\
\text { (Broda) }\end{array}$ \\
\hline $\begin{array}{l}\text { April } 26-46 \\
\text { Sun in nadir } \\
\text { Disappearance of the Pleiades } \\
\text { New moon } \\
\text { (convergence) }\end{array}$ & $\begin{array}{l}\text { Onset of harvest } \\
\text { Death of the sun, the moon, } \\
\text { the Pleiades, and Earth }\end{array}$ & $\begin{array}{l}\text { Cuzco } \\
\text { (Zuidema) }\end{array}$ \\
\hline $\begin{array}{l}\text { April } 19-22 \\
\text { Sun in nadir } \\
\text { Disappearance of the Pleiades } \\
\text { (heliacal set at dusk) } \\
\text { Next full moon } \\
\text { (convergence) }\end{array}$ & $\begin{array}{l}\text { Wet to dry season } \\
\text { crops } \\
\text { Feast to creator } \\
\text { god }\end{array}$ & $\begin{array}{l}\text { Huarochiri and } \\
\text { Coast of Peru } \\
\text { (Urton) }\end{array}$ \\
\hline
\end{tabular}

to examine the cosmological and astronomical context within which they occur. In the Andes, the reappearance of the Pleiades and the approach of the solstice in June announce a period of rebirth for the sun, the moon, the Pleiades, and the earth. What is the next cosmological sequence in Tenochititlan? In addition, do we find "symmetric opposition" in the activities of the mythic figures associated with the sun and the Pleiades in Aztec mythology? In Inca mythology, do the sun and the Pleiades have convergent roles or activities? These are further sets of questions that, once answered, would advance the study of the relationship between native astronomy and cosmology. We need to know the kind of metaphorical language used to express both opposed and convergent astronomical events.

Metaphors relate at least two phenomena together in figurative expression. The multiple interactions of celestial bodies that are observed in order to determine major transitions are better expressed in the language of metaphor, which expresses movement, transition, and reversibility or reflection. For example, the metaphors that come to mind from the papers in this conference include: weaving (Klein), hydraulics (HughJones, Richel-Dolmatoff, and Urton), the refraction of light in crystals (Reichel-Dolmatoff and Zuidema), and mirrors (Klein). Even the sym- 
bolic forms that at first glance seem to be static, in reality express dialectical concepts: the ushĩu (Zuidema), the serpent-mountain (Townsend), the caterpillar-jaguar (Hugh-Jones), and volcanoes (Coggins). I am reminded of the session in the Hayden Planetarium when Aveni asked Franklin to speed up the motion of the sky. The effect, as we watched the Milky Way change, was like watching a giant undulating snake move across the sky. We were able to perceive what the language of metaphor attempts to capture when apprehending the spatiotemporal relationships of tropical American cultures.

What we were attempting to capture in the planetarium was a dynamic process that somehow embodies temporal order. The ancient astronomers of tropical America attempted to capture these same processes of structural order in settlement plans and architectural and ritual space. We might label their attempt relational, or dialectical geometry. The paper by Aveni and Hartung illustrates the concern that Mesoamericans had for obligating the perceiver to focus upon the interaction of celestial forces by constructing the environment of ceremonial space around the principle of what Aveni and Hartung have called interbuilding relationships. I think that the astronomer-architect-priests were attempting to assure that the dynamic (or dialectical) perspective they had discovered would be maintained from generation to generation. Likewise, the ceque lines of Cuzco, which embody the organizational principle of dialectical, reversible dualism, are a means of perpetuating the epistemological discovery that there exists a dynamic relationship between time and space. For example, Dearborn and White's paper confirms Zuidema and Urton's arguments concerning the relationship between the zenith solar passage, the path of the Pleiades (its heliacal rise and set), and the June solstice. The construction of the Torreón obligates the observer to adopt a perspective that focuses upon the relation of one transition of time to another instead of pinpointing the precise date of an astronomical event. I would suggest that such a dynamic perspective is shared by the cultures of the American tropics as culture confronts nature in the reversible world of the tropics. Through the application of dialectical logic, the cosmologies of these cultures construe the contradictions perceived in nature as necessarily interdependent. The contradictions can never be resolved because cosmic order is maintained by the dialectical tension resulting from the reversible relationship pertaining between opposed elements. 


\section{ACKNOWLEDGMENTS}

I would like to give special thanks to my husband, W.H. Isbell, and Lauris McKee for reading numerous versions of this paper and making helpful comments.

\section{NOTES AND REFERENCES}

1. C. Levi-Strauss, The Savage Mind (Chicago: University of Chicago Press, 1966), chap. 1. "The Science of the Concrete."

2. RoY WAGNER, The Invention of Culture (Chicago: University of Chicago Press, 1975), chap. 3, p. 52 .

3. I am using the term schemata in the sense of U. Neisser, Cognition and Reality: Principles and Implications of Cognitive Psychology (San Francisco: W. H. Freeman, 1976).

4. A.F. Aveni, Skywatchers of Ancient Mexico (Austin: University of Texas Press, 1980), "Astronomy with the Naked Eye," chap. 3, pp. 48-132.

5. G. Urton, "The Use of Native Cosmologies in Archaeoastronomical Studies: The View from South America," in Archaeoastronomy in the Americas, ed. R. Williamson (Santa Barbara, Calif.: Ballena Press, in press).

6. Aveni. ${ }^{3}$

7. Aveni ${ }^{3}$ p. 63.

8. A.F. Aveni and G. Urton, "A Preliminary Investigation of Geometrical and Astronomical Order in the Nazca Lines," unpublished ms.

9. R.T. Zuidema, "Organizing Space for Computing the Calendar," unpublished ms.

10. R.T. Zuidema, "The Inca Observations in Cuzco of the Solar and Lunar Passages through Zenith and Antizenith," in Archaeoastronomy in the Americas, ed. R. Williamson (Santa Barbara, Calif.: Ballena Press, in press).

11. A.F. Aveni, "Tropical Archaeoastronomy," Science, vol. 213 (1981), pp. 161-71.

12. Aveni. ${ }^{10}$

13. Aveni, ${ }^{3}$ pp. 294-311, and Zuidema. ${ }^{9}$

14. J. Sherbondy, "Water in the Ritual Calendar of Cuzco," paper presented at the symposium entitled Myth and Ritual in Andean Societies at the annual American Anthropological Association meeting, Washington, D.C., 1980. 